\title{
Highly Fluorescent Dye-Doped Silica Nanoparticles Increase Flow Cytometry Sensitivity for Cancer Cell Monitoring
}

\author{
M. -Carmen Estévez, Meghan B. O’Donoghue, Xiaolan Chen, and Weihong Tan ( $\square)$ \\ Center for Research at the Bio/Nano Interface, Departments of Chemistry and Department of Physiology and Functional Genomics, \\ Shands Cancer Center and UF Genetics Institute, McKnight Brain Institute, University of Florida, Gainesville, Florida 32611-7200, USA \\ Received: 18 January 2009 / Revised: 12 March 2009 / Accepted: 16 March 2009 \\ (C)Tsinghua University Press and Springer-Verlag 2009. This article is published with open access at Springerlink.com
}

\begin{abstract}
Early and accurate diagnosis and treatment of cancer depend on rapid, sensitive, and selective detection of tumor cells. Current diagnosis of cancers, especially leukemia, relies on histology and flow cytometry using single dye-labeled antibodies. However, this combination may not lead to high signal output, which can hinder detection, especially when the probes have relatively weak affinities or when the receptor is expressed in a low concentration on the target cell surface. To solve these problems, we have developed a novel method for sensitive and rapid detection of cancer cells using dye-doped silica nanoparticles (NPs) which increases detection sensitivity in flow cytometry analyses between 10- and 100-fold compared to standard methods. Our NPs are $\sim 60 \mathrm{~nm}$ in size and can encapsulate thousands of individual dye molecules within their matrix. We have extensively investigated surface modification strategies in order to make the NPs suitable for selective detection of cancer cells using flow cytometry. The NPs are functionalized with polyethylene glycol (PEG) to prevent nonspecific interactions and with neutravidin to allow universal binding with biotinylated molecules. By virtue of their reliable and selective detection of target cancer cells, these NPs have demonstrated their promising usefulness in conventional flow cytometry. Moreover, they have shown low background signal, high signal enhancement, and efficient functionalization, either with antibody- or aptamer-targeting moieties.
\end{abstract}

\section{KEYWORDS}

Silica nanoparticle, flow cytometry, aptamer, cell detection, polyethylene glycol, fluorophore

\section{Introduction}

Flow cytometry has become a common and indispensable tool in many clinical and research settings. For example, it is routinely used to monitor CD4 and CD8 T-cell counts in order to track HIV disease progression [1] and evaluate B-cell lymphoid neoplasms [2]. More sophisticated tasks are nevertheless still challenging. Thus, flow cytometry-based assays for reliable identification of (1) rare residual cell populations that remain after treatment, (2) rare sloughed off cancer cells useful for early disease detection, and (3) cell populations that have low levels of target protein expression [3] have been developed, although when conventional flow cytometers are used such assays are not feasible. Commonly used fluorescent imaging probes, such as organic and bioluminescent-encoded fluorophores

Address correspondence to tan@chem.ufl.edu 
or fluorescent proteins, are often employed for these applications. Moreover, the widespread use of flow cytometry in clinics is in part a consequence of the growing number of fluorophores that have been developed in an effort to solve problems such as sensitivity and multicolor assays. On the other hand, flow cytometry has also become a routinely used technique for a variety of biological applications, besides clinical diagnosis, which rely on the detection of surface receptors. Nevertheless, some of the current fluorescent probes exhibit weak fluorescence signals and low photobleaching thresholds and stability because of their intrinsic photophysical properties and therefore their use is somewhat limited for the purposes indicated above. Moreover, early diagnosis of disease is often contingent on the sensitivity of the tools used for the detection and monitoring of smaller and less abundant targets, such as individual cells, proteins, and surface sugars. Consequently, these tools must successfully integrate nanotechnological materials and methods with biological systems in order to achieve the detection sensitivity required for such biomedical applications [4].

To address these challenges, fluorophore-doped, bright, and photostable silica NPs have recently been used as novel substrates for optical signaling [5]. Dyedoped silica NPs possess several useful features: they are small, bright, stable, biocompatible, hydrophilic, chemically inert, easily separated via centrifugation, and not subject to microbial attack. Single dyedoped silica NPs have previously been used for ultrasensitive DNA analysis down to sub-fmol/L concentrations [6]. The high brightness of the NPs has also allowed sensitive cell detection by conventional fluorescence spectroscopy and microscopy $[7,8]$ and single bacterium monitoring within $20 \mathrm{~min}$ [9]. Each NP encapsulates hundreds, or even thousands, of dye molecules in a $\sim 60 \mathrm{~nm}$ silica sphere. Therefore, even considering the likely quenching between the closely packed molecules inside the matrix, an overall fluorescence between two and three orders of magnitude larger than that from individual dyes is usually achieved, thus providing an extremely strong fluorescence signal for ultrasensitive bioanalysis.

In addition, these NPs can be biofunctionalized with a wide array of different molecular recognition probes, such as antibodies or aptamers. Aptamers are single-stranded DNA or RNA oligonucleotides which selectively bind their target with high affinity and specificity. They are selected using a combinatorial approach called SELEX (Systematic Evolution of Ligands by EXponential enrichment). This process involves repeated rounds of in vitro selection with a starting pool of DNA or RNA and the molecular target, such as a small molecule, protein, or even whole living cells [10,11]. We hypothesized that the capabilities of flow cytometry could be complemented, and possibly greatly enhanced, by combining the selectivity of aptamers and antibodies with the high signal enhancement and ease of functionalization offered by dye-doped silica NPs. This would require a probe capable of universally tagging the target of interest, one that could sensitively and reliably identify the types of rare events noted above, and one not limited by the modest signal enhancement of currently available flow cytometry probes.

Commercial fluorescent beads with sizes ranging from several hundreds of nanometers to a few micrometers are commonly used in flow cytometry. Their main function is in instrument calibration. However, since their size is relatively large, they are incompatible for use as labeling probes for cell detection using flow cytometry. Smaller commercial probes with the high brightness and surface compatibility required for further bioconjugation are available, but no results reporting any improvement in sensitivity can be found in the current literature. This indicates that the implementation of these nanoprobes in flow cytometry analysis has most likely been blocked by the complicated issues of nonspecific adsorption. Because of their multiplexing capabilities, small fluorescent nanoprobes, such as quantum dots, have been reported to be potentially useful in sophisticated polychromatic flow cytometers for immunophenotyping, but the enhancement of sensitivity has also not been addressed in this case [12]. It appears that the potential of fluorescent NPs for the purpose of enhancing the signal of flow cytometers has not yet been demonstrated. Therefore, to the best of our knowledge, this is the first report specifically focused on applying fluorescent dye- 
doped silica NPs with the aim of increasing the signal in conventional flow cytometry instruments. The results presented here suggest the potential usefulness of fluorescent silica NPs as complementary fluorophores when sensitivity can become a limiting factor in conventional flow cytometry analyses.

\section{Experimental}

\subsection{Materials}

Tetramethylorthosilicate (TEOS), aminopropyltriethoxysilane (APTES), 3-trihydroxysilylpropylmeth ylphosphonate (THPMP), and tris(2,2'-bipyridyl)dichlororuthenium(II) hexahydrate (Rubpy) dye were obtained from Sigma-Aldrich (St. Louis, MO). Fluorescein-5-isothiocyanate (FITC) was purchased from Invitrogen (Carlsbad, CA). Polyethylene glycol (PEG) $(\mathrm{MW}=5000)$ functionalized with biotin at one end and $\mathrm{N}$-hydroxysuccinimide at the other end (NHS-PEG 5000 $-\mathrm{B}$ ) was purchased from Nektar (San Carlos, CA). Neutravidin was obtained from Pierce Biotechnology, Inc. (Rockford, IL). The pure monoclonal anti-PTK7 antibody, which recognizes human protein tyrosine kinase-7, and the Biotinylation Kit used to label the antibody were purchased from Miltenyi Biotec, Inc. (Auburn, CA).

\subsection{Cell lines}

CCRF-CEM human acute lymphoblastic leukemia cells (CCL-119 T-cell), Ramos human Burkitt's lymphoma cells (CRL-1596 B-cell), HeLa cervical cancer cells (CCL-2), NB-4 and HL-60 acute promyelocytic leukemia cells were obtained from the ATTC cultures. The cells were grown in pH 7.4 RMPI 1640 medium supplemented with 10\% FBS (fetal bovine serum) (heat-inactivated, GIBCO) and $100 \mathrm{IU} / \mathrm{mL}$ penicillin/ streptomycin (Cellgro). In each experiment, cells were washed before and after incubation with the washing buffer made of $4.5 \mathrm{~g} / \mathrm{L}$ glucose and $5 \mathrm{mmol} / \mathrm{L} \mathrm{MgCl}_{2}$ in Dulbecco's phosphate buffered saline with calcium chloride and magnesium chloride (Sigma). The binding buffer used for aptamer and NP incubation was prepared by adding yeast tRNA $(0.1 \mathrm{mg} / \mathrm{mL})$ (Sigma) and BSA (1 mg/mL) (Sigma) into washing buffer to reduce the nonspecific binding.

\subsection{Preparation and characterization of dye-doped silica NPs}

Fluorescein-doped silica NPs (NP(FITC)) were prepared by the Stöber method using the following procedure: FITC $(2.75 \mu \mathrm{mol})$ was dissolved in $500 \mu \mathrm{L}$ of anhydrous DMSO and APTES was added at a molar ratio of 1.2:1 APTES:dye. The APTES was allowed to react with the dye for $1 \mathrm{~h}$ in the dark, while shaking, prior to the synthesis of the NPs. Glass reaction tubes were washed with $1 \mathrm{~mol} / \mathrm{L} \mathrm{NaOH}$ solution for 15 min and rinsed with deionized water and ethanol in order to prevent the seeding of the NPs on the glass surface. EtOH (16.75 mL) and $\mathrm{NH}_{4} \mathrm{OH}(1.275 \mathrm{~mL})$ were added to the tubes. Under continuous stirring, the solution of dye-APTES conjugate was added to the mixture and left to react for $1 \mathrm{~h}$. Next, TEOS $(710 \mu \mathrm{L})$ was added to the mixture, and the reaction was allowed to proceed for $24 \mathrm{~h}$ protected from light while continuously stirring. The particles were recovered by centrifugation at $14,000 \mathrm{rpm}(20,000 \mathrm{~g})$ for $30 \mathrm{~min}$. The particles were washed three times with $\mathrm{EtOH}$ and three times with deionized water to remove any free dye or unbound molecules, and they were finally resuspended in deionized water. The size of the NPs was determined by transmission electron microscopy (TEM, Hitachi H7100, Tokyo, Japan) and was found to be $67.1 \mathrm{~nm}$ $\pm 6.0 \mathrm{~nm}$ after measuring the size of more than 100 particles. The fluorescence of the NPs was determined by comparing the fluorescence signal of the NP solution with a calibration curve of the pure dye under the same conditions. The average fluorescence of the NPs was equivalent to 4300 dye molecules/NP. To introduce amino groups on the surface of NPs, $10 \mathrm{mg}$ of NP(FITC) was diluted to a total volume of $500 \mu \mathrm{L}$ of PBS (10 mmol/L, pH 7.4), and APTES $(12.5 \mu \mathrm{L})$ and THPMP $(37.5 \mu \mathrm{L})$ were then added to the solution together with $\mathrm{NH}_{4} \mathrm{OH}(20 \mu \mathrm{L})$. The reaction was stirred overnight, and the particles (NP(FITC)- $\mathrm{NH}_{2}$ ) were washed as described above and reconstituted in PBS $(10 \mathrm{mmol} / \mathrm{L}, \mathrm{pH} 7.4)$ to a concentration of $10 \mathrm{mg} / \mathrm{mL}$.

Rubpy dye-doped silica NPs (NP(Rubpy)) were prepared by the reverse microemulsion method as follows: Triton X-100 (1.77 mL), cyclohexane (7.5 
$\mathrm{mL})$, and $n$-hexanol $(1.6 \mathrm{~mL})$ were added to a $20 \mathrm{~mL}$ glass vial followed by $80 \mu \mathrm{L}$ of a solution of Rubpy dye in deionized water $(90 \mathrm{mmol} / \mathrm{L})$. Next, $400 \mu \mathrm{L}$ of deionized water and $100 \mu \mathrm{L}$ of TEOS were added, and the reaction was stirred for $30 \mathrm{~min}$. To initiate silica polymerization, $60 \mu \mathrm{L}$ of $\mathrm{NH}_{4} \mathrm{OH}$ was added to the mixture, and the reaction was allowed to proceed for $15 \mathrm{~h}$. The microemulsion was then broken up by adding acetone, and the suspension was centrifuged and washed as described above. The size of the NPs was determined by TEM and was found to be $44.9 \mathrm{~nm} \pm 2.9 \mathrm{~nm}$ after measuring the size of more then 100 particles. The fluorescence of the NPs was determined by comparing the fluorescence signal of the NP solution with a calibration curve of the pure dye under the same conditions. The average fluorescence of the NPs was equivalent to 15,000 dye molecules/NP. The amino surface modification was performed by adding $50 \mu \mathrm{L}$ of TEOS and $50 \mu \mathrm{L}$ of $\mathrm{NH}_{4} \mathrm{OH}$ to a suspension of $10 \mathrm{mg} / \mathrm{mL}$ of the NPs in PBS followed by $20 \mu \mathrm{L}$ of APTES and $30 \mu \mathrm{L}$ of THPMP after $30 \mathrm{~min}$. The reaction was left to proceed for $8 \mathrm{~h}$, and the particles were washed as described above. The particles (NP(Rubpy)- $\mathrm{NH}_{2}$ ) were resuspended in PBS buffer to a final concentration of $10 \mathrm{mg} / \mathrm{mL}$. The zeta potential was measured on a Brookhaven PALS Zeta Potential Analyzer, version 3.16, using the Smoluchowski model. Samples with each different coating modification were analyzed at a concentration of $\sim 120 \mu \mathrm{mol} / \mathrm{L}$ at $23{ }^{\circ} \mathrm{C}$, with an average of 10 runs for each sample.

\subsection{Surface modification of amino-functionalized NPs with neutravidin}

$1 \mathrm{mg}$ of the amino-functionalized NPs was diluted in $500 \mu \mathrm{L}$ of PBS, and a fresh solution of NHS-PEG ${ }_{5000}-\mathrm{B}$ (500 $\mu \mathrm{L}$ of a solution of $10 \mathrm{mg} / \mathrm{mL}$ ) was added to the suspension. The reaction was stirred for $2 \mathrm{~h}$, and excess reagent was removed by centrifuging and washing the NPs three times with PBS. The particles were reconstituted in PBS $(500 \mu \mathrm{L})$, and the subsequent coating of the particles with the neutravidin was performed through the free biotin group present in the $\mathrm{PEG}_{5000}$ molecule by direct incubation with a solution of neutravidin in PBS (500 $\mu \mathrm{L}$ of a solution of $4 \mathrm{mg} / \mathrm{mL}$ ). The reaction mixture was gently shaken overnight at $4{ }^{\circ} \mathrm{C}$. The NPs were then washed with PBS to remove the free neutravidin as described above and resuspended in PBS buffer to a final concentration of $1 \mathrm{mg} / \mathrm{mL}$ and stored at $4{ }^{\circ} \mathrm{C}$ for up to 2 months.

\subsection{DNA synthesis and labeling}

All the aptamer sequences had been previously selected for specific cancer cell lines. Both the FITC-labeled and biotin-labeled aptamers were synthesized in house using an ABI3400 DNA/RNA synthesizer (Applied Biosystems, Foster City, CA). The dye-labeled aptamers were prepared with fluorescein at the 3'-end by using 3'-(6-fluorescein) CPG (controlled pore glass). The biotin-labeled aptamers were synthesized using 3'-biotin-CPG, and all of them had an additional linker between the biotin and the specific sequence of either a poly $\mathrm{T}$ (10 $\mathrm{T}$ bases, Poly $_{10}$ ) or a spacer consisting of 3 units of hexaethylene glycol (18 extra units of ethylene oxide). The aptamer sgc8 used for confocal microscopy experiments consisted of a Cy5 label at the $5^{\prime}$-end and a poly $\mathrm{T}_{10}$-biotin at the $3^{\prime}$-end $\left(\mathrm{Cy} 5\right.$-sgc8-PolyT ${ }_{10^{-}}$ Biotin). All fabricated DNA was purified by reverse phase high-pressure liquid chromatography (HPLC) using a ProStar HPLC (Varian, Walnut Creek, CA) with a C18 column (Econosil, $5 \mu \mathrm{m}, 250 \mathrm{~mm} \times$ $4.6 \mathrm{~mm}$ ) from Alltech (Deerfield, IL). A Cary Bio-300 UV spectrometer (Varian, Walnut Creek, CA) was used to measure absorbances to calculate aptamer concentration. All oligonucleotides were synthesized by solid-state phosphoramidite chemistry at a $1-\mu \mathrm{mol}$ scale. The completed sequences were then deprotected in AMA (ammonium hydroxide/40\% aqueous methylamine $1: 1$ ) at $65{ }^{\circ} \mathrm{C}$ overnight and further purified by HPLC using a C18 column.

\subsection{Preconjugation of aptamer to the NPs}

The aptamer was immobilized on the surface of the NPs by incubating the NPs with the biotinylated aptamer (1:1000 molar ratio) in PBS $10 \mathrm{mmol} / \mathrm{L}$ buffer ( $\mathrm{pH}$ 7.4) under gentle shaking overnight at $4{ }^{\circ} \mathrm{C}$. The conjugates were washed three times with PBS buffer and finally reconstituted in binding buffer and stored at $4{ }^{\circ} \mathrm{C}$ until use. 


\subsection{Flow cytometry experiments}

Flow cytometry analyses were performed in a FACScan cytometer equipped with CellQuest Software (Becton Dickinson Immunocytometry Systems, San Jose, CA) by counting 30,000 events, and the data were analyzed using WinMDI software. The binding experiments were performed as follows: cells $\left(5 \times 10^{5}\right)$ were washed twice with washing buffer $(500 \mu \mathrm{L})$ by centrifugation at $950 \mathrm{rpm}$ for $4 \mathrm{~min}$ and reconstituted in $100 \mu \mathrm{L}$ of binding buffer. Aptamer labeled with dye (aptamer-FITC) or with biotin (aptamer-biotin) was added $(100 \mu \mathrm{L}, 125 \mathrm{nmol} / \mathrm{L})$ and incubated with the cells for $20 \mathrm{~min}$ at $4{ }^{\circ} \mathrm{C}$. Cells were washed twice and reconstituted in binding buffer $(200 \mu \mathrm{L})$. Cells with aptamer-FITC were directly detected by flow cytometry. For cells with aptamerbiotin, NPs were then added (10 $\mu \mathrm{L}$ of a solution of $1 \mathrm{mg} / \mathrm{mL}$, unless specified otherwise) and were incubated for $10 \mathrm{~min}$ at $4{ }^{\circ} \mathrm{C}$. Cells were washed twice and finally suspended in $200 \mu \mathrm{L}$ of binding buffer for the detection. Fluorescein-labeled unselected ssDNA library was used as negative control for FITC-labeled aptamers, whereas a nonspecific aptamer labeled with biotin was used as a control for the NP experiments.

\subsection{Confocal imaging}

Fluorescence imaging was conducted on a confocal microscope setup consisting of an Olympus IX81 inverted microscope with an Olympus Fluoview 500 confocal scanning system and three lasers, a tunable argon ion laser $(458,488,514 \mathrm{~nm})$, a green $\mathrm{He}-\mathrm{Ne}$ laser $(543 \mathrm{~nm})$, and a red $\mathrm{He}-\mathrm{Ne}$ laser $(633 \mathrm{~nm})$ with three separate photomultiplier tubes for detection. The cellular images were taken with a $20 \times 0.70$ numerical aperture (NA) objective. The fluorescent NPs were excited with the $488 \mathrm{~nm}$ line of the argon ion laser, and emission was detected using a 505-525 $\mathrm{nm}$ bandpass filter for the NP(FITC) and a $610 \mathrm{~nm}$ long-pass filter for NP(Rubpy). Cy5-labeled aptamer was excited at $633 \mathrm{~nm}$ with $\mathrm{HeNe}$ laser, and the emission was detected with a $660 \mathrm{~nm}$ long-pass filter.

\section{Results and discussion}

The main aim of this work is the development of highly fluorescent, selective, and reproducibly synthesizable probes based on dye-doped silica NPs as high-amplification signaling elements and to implement them as alternative fluorescent probes in flow cytometry analyses. However, nanoparticlebased bioassays can be strongly influenced by their tendency to aggregate, causing nonspecific adsorption. This problem can be especially severe when DNA oligonucleotides, such as aptamers, which are negatively charged, are used as specific ligands, and the target surface is the complex and also charged cell membrane. Under these conditions, the choice of an appropriate surface modification for the NPs combined with a generic and simple ligand immobilization is essential to the development of a suitable and reproducible methodology.

Accordingly, plain NPs were initially prepared using two previously established methodologies, the Stöber method [13] and the reverse microemulsion method. We initially investigated several different covalent strategies to functionalize the silica surface [14], including carboxylic groups (-COOH) [15], thiol groups $(-\mathrm{SH})[16]$ and amino groups $\left(-\mathrm{NH}_{2}\right)$ [15]. Different organic bifunctional linkers were also tested to couple the NPs with functionalized amine (aptamer- $\mathrm{NH}_{2}$ ). Unfortunately, under all these conditions, severe nonspecific adsorption of the particles onto the cell surface was observed, even without the aptamer (data not shown). Therefore, to prevent nonspecific interactions, we next considered coupling the NPs with a PEG linker which is known to improve biocompatibility for both in vivo and in vitro applications by enhancing hydrophilicity [17-19]. As expected, a considerable decrease in nonspecific interaction was observed, even though the accompanying shift in the fluorescence signal was still minimal (data not shown). Since the use of proteins can also enhance the hydrophilicity of the probes, thereby minimizing adverse nonspecific interactions, a coating of avidin was also added to the surface of the NPs, either by covalently binding amino groups of the protein to carboxy-modified NPs or by physical adsorption onto plain NPs. In both cases, significant binding of the particles was observed, even in the absence of aptamers. This result could be explained by the overall positive charge that avidin has (pI 9.5-10), which makes it especially 
prone to nonspecific binding. On the other hand, an obvious advantage of using avidin is the convenience of exploiting the avidin-biotin interaction by introducing the biotin group in the aptamer. The interaction is exceptionally strong and stable, similar in strength to a covalent bond, and occurs relatively fast under mild conditions, thus allowing a more versatile design for labeling and detecting cancer cells by flow cytometry. Based on these preliminary findings, surface modification of the NPs was finally achieved by combining both a PEG moiety and a subsequent protein surface modification with neutravidin, which is almost neutral (pI 6.3) (Fig. 1).

Plain fluorescent silica NPs were prepared using two different dyes, FITC and Rubpy. Both have been proven to be efficient fluorophores which can be trapped inside the silica matrix. Furthermore, they show excitation and emission spectra which meet the specifications of most flow cytometers. Thus, in single laser instruments, they can be excited with the argon laser $(\lambda=488 \mathrm{~nm}$ ) and can be monitored in two independent emission channels (FL1 for FITC $\left(\lambda_{\text {exc(FITC) }}=488 \mathrm{~nm} ; \lambda_{\text {em(FITC) }}=520 \mathrm{~nm}\right)$ and FL3 for Rubpy $\left.\left(\lambda_{\text {exc(Rupby) }}=450 \mathrm{~nm} ; \lambda_{\text {em(Rubpy) }}=600 \mathrm{~nm}\right)\right)$. FITC is an organic fluorophore with mainly hydrophobic behavior. The most effective way to trap it inside the particles is by performing an initial covalent preconjugation of the dye with an organosilane which incorporates an appropriate functional group [20]. This methodology has been shown to be efficient in covalent trapping of other organic fluorophores besides FITC, such as rhodamine dyes [21]. Thus, in this case, the FITC was covalently bound to APTES through the isothiocyanate group in the dye and the amino group of the silane. This precoupling step is necessary in order to avoid the leakage of the dye during the manipulation of the NPs. In the case of the inorganic dye Rubpy, which possesses a mainly hydrophilic nature, the reverse microemulsion method leads to a more efficient dye encapsulation with no dye leakage during subsequent handling of the NPs [22].

In both cases, uniform, spherical, and welldispersed NPs in the nanometer range were obtained $(44.9 \mathrm{~nm} \pm 2.9 \mathrm{~nm}$ for NP(Rubpy) and $67.1 \mathrm{~nm} \pm 6.0$ $\mathrm{nm}$ for NP(FITC)) (Fig. 1). After successive washes with both ethanol and water no leakage of dyes was observed by determining the fluorescence of the supernatant before and after centrifugation of the NPs (data not shown). Although the determination of an accurate number of dye molecules trapped inside the NPs is not possible, because of the potential effect of self-quenching of dye molecules in close proximity, it is possible to determine the average fluorescence of the NPs compared with pure dyes. Therefore, calibration curves with the pure fluorophore were performed in order to elucidate the equivalent number of dye molecules trapped inside the matrix. Figure 1(b) shows an example of the fluorescence emission spectra of the NPs and the pure dye (for both NP(Rubpy) and NP(FITC). The amplification of the fluorescence signal obtained with the NPs can be clearly seen (an NP(FITC)) solution of concentration $0.85 \mathrm{nmol} / \mathrm{L}$ shows a fluorescence around four times more intense than a pure FITC solution of concentration $500 \mathrm{nmol} / \mathrm{L}$ ). By interpolation of the fluorescence values into the calibration curve the average fluorescence intensity of both NPs was determined. NP(FITC) had an average fluorescence intensity equivalent to $\sim 4300$ dye molecules / NP whilst the value for NP(Rubpy) was $\sim 15,000$ dye molecules/NP. The fluorescence shown by both NPs was therefore extremely high compared with pure fluorophores, whereas the size of the NPs was small enough $(<100 \mathrm{~nm})$ (see Fig. 1) for their individual detection using flow cytometry. This is especially important since cytometers are typically able to detect particles and cells in the size range $0.5 \mu \mathrm{m}$ to $40 \mu \mathrm{m}$ in diameter. In fact, it is precisely because of size similarities between the species that fluorescent microbeads in the micrometer range are commonly used in flow cytometry, albeit for their own detection or calibration purposes rather than cell detection. To create the conditions required to monitor species smaller than cells, the instrument's laser parameters can, under certain circumstances, be optimized to detect particles smaller than $100 \mathrm{~nm}$, such as viruses [23], even though bigger particles, such as cells, are still going to remain undetectable. Hence, the smaller dimensions of the fluorescent NPs ensure (1) the absence of fluorescence interference coming from the detection of free unbound NPs with the flow cytometer under conditions otherwise required for

\section{包 Springer}




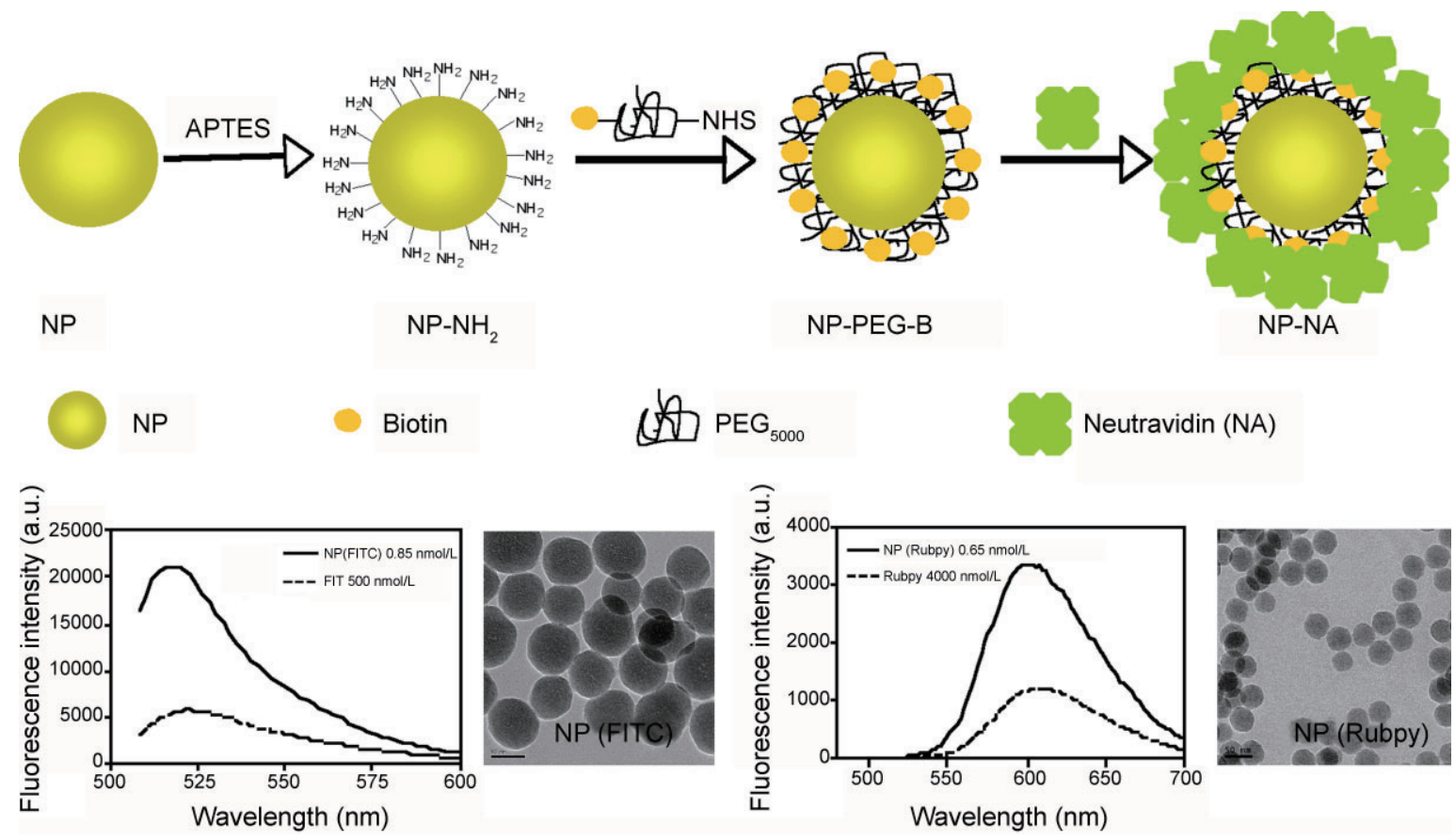

Figure 1 Fluorescent dye-doped silica NPs: (a) scheme illustrating the surface modification of the fluorescent silica NPS with PEG and neutravidin; (b) fluorescence signal amplification obtained with NPs and TEM images of NP(FITC) and NP(Rubpy). The concentration of NPs was calculated from the size and the average silica density. The scale bar represents $50 \mathrm{~nm}$

detecting cells and (2) the possibility of using them as fluorescent probes to label cells.

Both NPs were modified with amino groups on the surface by adding APTES together with methyl phosphonate groups using THPMP. The amino groups allow covalent immobilization of the NPs to the NHS group present on the PEG linker (NHS$\mathrm{PEG}_{5000}$-Biotin), while the phosphonate group helps to avoid aggregation of the NPs. Thus, whereas bare silica presents negative values of zeta potential, the incorporation of a high concentration of amino groups on the surface leads to a higher zeta potential (more positive values) which, in turn, results in higher aggregation of the NPs and less dispersed suspensions [15]. On the other hand, the addition of an inert functional group, the methyl phosphonate, which is negatively charged, decreases the zeta potential, resulting in a stronger repulsion between the particles, and this reduces the aggregation. Therefore, two different mixtures, APTES/THPMP (ratio 25:75 v/v) for NP(FITC) and APTES/THPMP / TEOS (ratio (20:30:50 v/v) for NP(Rubpy), were used for the addition of the functionalized layer. The zeta potential of the NPs was determined after each surface modification, and the results are summarized in Table 1. Plain particles have negative zeta potentials of around -30 to $-40 \mathrm{mV}$. NP(FITC) clearly increased this to less negative values $(-9 \mathrm{mV})$, even with the presence of phosphonate groups in the copolymerized moiety. In contrast, we found a more negative zeta potential for NP(Rubpy), which resulted from the presence of additional TEOS in the mixture. When the PEG linker was covalently coupled through the NHS group to the amino functionalization on the NPs, a similar zeta potential value was observed in both particles (around -14 to

Table 1 Zeta potential measurements of silica NPs with different coating functionalization

\begin{tabular}{cc|c|c}
\hline NP type & Surface moiety & $\begin{array}{c}\mathrm{NP}(\mathrm{FITC}) \\
\zeta(\mathrm{mV})\end{array}$ & $\begin{array}{c}\mathrm{NP}(\mathrm{Rubpy}) \\
\zeta(\mathrm{mV})\end{array}$ \\
\hline $\mathrm{NP}$ & None (plain NP) & $-41 \pm 1.0$ & $-33 \pm 0.9$ \\
$\mathrm{NP}-\mathrm{NH}_{2}$ & $\mathrm{NH}_{2} / \mathrm{P}\left(\mathrm{CH}_{3}\right) \mathrm{O}_{2}^{-}$ & $-9 \pm 1.3$ & $-41 \pm 0.7$ \\
$\mathrm{NP}-\mathrm{PEG}-\mathrm{B}$ & $\mathrm{PEG}_{5000}$ & $-18 \pm 1.0$ & $-14 \pm 0.8$ \\
$\mathrm{NP}-\mathrm{NA}$ & Neutravidin & $-20 \pm 0.9$ & $-11 \pm 1.2$ \\
$\mathrm{NP}-\mathrm{Apt}$ & Aptamer & $-23 \pm 1.7$ & $-21 \pm 0.8$ \\
\hline
\end{tabular}


$-18 \mathrm{mV}$ ), which indicates efficient surface coverage. Neutravidin was then added to the NPs coated with biotinylated PEG to allow conjugation with the biotinylated aptamer or antibody.

Next, in order to study the behavior of the NPs, different aptamer sequences were tested and analyzed by flow cytometry. Using cell-based SELEX, the aptamers tested have all been previously selected for the recognition of surface receptors on the membranes of cancer cells [11, 24]. The cells were first incubated with the aptamers, allowing them to bind their specific target. After washing the cells to remove the unbound aptamer, the NPs were added and incubated for a short period of time (10 $\mathrm{min}$ ) (see Fig. 2). All the aptamers consisted of the specific aptamer sequence, an additional linker, either a hydrophilic segment of ethylene glycol or a sequence of 10 bases of thymine ( PolyT $_{10}$ ), and, finally, the biotin group at the 3 '-end. The linkers were optimized to allow enough flexibility to ensure that the biotinneutravidin interaction did not interfere with the correct aptamer folding. Following this strategy, it was possible to (1) study and optimize the influence of the silica NPs on the final signal monitored by flow cytometry in order to minimize the potential nonspecific adsorption and maximize the mean fluorescence signal and (2) study the feasibility of these probes with a wide variety of aptamer sequences to show their broad-based utility.

To demonstrate the effectiveness of the nanoconjugate system, the aptamer sgc8 was initially tested using NP(FITC), and the mean fluorescence signal was compared with the one obtained with the commonly used single fluorophore-labeled aptamer (sgc8-FITC). This aptamer selectively binds acute lymphoblastic leukemia CEM cells with a high affinity $\left(K_{\mathrm{d}} \sim 0.80 \mathrm{nmol} / \mathrm{L}\right)$. Under saturation conditions of the specific surface cell receptor for $\operatorname{sgc} 8$ [25], an average maximum fluorescence signal of around 13 is achieved $(13.7 \pm 1.5)$. As can be seen in Fig. 3, a large fluorescence signal enhancement (SE) was observed using $2.5 \mu \mathrm{g}$ of NPs (mean fluorescence signal of $168.6 \pm 69.5$ ). Preliminary results obtained in our lab suggest that the number of specific receptors for the sgc8 aptamer on the cell surface is around $10^{5}$ (unpublished results). Based on the size and amount of NPs, as indicated above, with the average density of the silica at $1.96 \mathrm{~g} / \mathrm{mL}$, around $8 \times 10^{9} \mathrm{NPs}$ were added. Considering the number of cells used in each experiment $\left(5 \times 10^{5}\right)$, this resulted in a slight default in the ability of NPs to cover all the receptors on the cell. In this case, coverage was sufficient to observe a high fluorescence signal, but steric hindrance between the NPs is still expected to result in an incomplete coverage of the cell surface with the NPs. Under such conditions, NPs with a size of about $60 \mathrm{~nm}$ will sterically hinder interaction with the aptamers if they are bound to receptors in close proximity. Not withstanding this limitation, we found that increasing the amounts of NPs $(5 \mu \mathrm{g}$ and $20 \mu \mathrm{g})$ led to an increase in the mean signal (see Fig. 3), thus confirming partial coverage. As indicated in Table 2, the SE gradually increased as more NPs were added, reaching an enhancement of almost two orders of magnitude when $20 \mu \mathrm{g}$ of NPs $\left(6.4 \times 10^{10} \mathrm{NPs}\right)$ were added. Higher amounts of NPs led to a saturation of the signal; therefore, a final amount of NPs, between 5 and $20 \mu \mathrm{g}$, was used in different combinations.

As shown in Fig. 3, the signal from the NPs incubated with either target or negative cells, but in the absence of aptamers, was negligible, even with the higher amount of NPs. This indicates that the use of

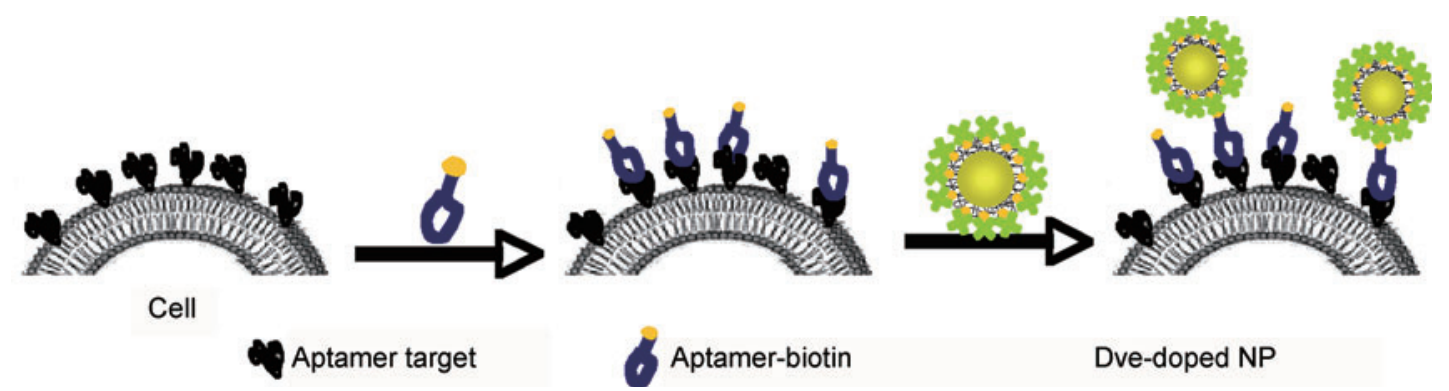

Figure 2 Two-step incubation procedure of the fluorescent NPs with cells. The cells are incubated with the biotinylated aptamer, washed and incubated with the neutravidin-coated NPS 
Table 2 Signal enhancement using dye-doped silica NPs with different cell / aptamer combinations

\begin{tabular}{|c|c|c|c|c|c|c|c|c|}
\hline \multirow{2}{*}{ Cell } & \multirow{2}{*}{ Aptamer } & \multirow{2}{*}{ Aptamer ${ }^{a}$} & \multirow{2}{*}{$N P(\mu) g^{b}$} & \multicolumn{4}{|c|}{ Mean fluorescence, $F$} & \multirow{2}{*}{$\begin{array}{c}\text { Signal } \\
\text { enhancement } \\
(S E)^{c}\end{array}$} \\
\hline & & & & $A p t+N P(F I T C)$ & Aptamer-FITC ${ }^{a}$ & NP(FITC) & Lib-FITC & \\
\hline \multirow[t]{4}{*}{ CEM } & $\operatorname{sgc} 8$ & sgc8-poly $T_{10}-B$ & 2.5 & $168.6 \pm 69.5$ & $13.7 \pm 1.5$ & $3.90 \pm$ & $4.13 \pm 0.33$ & $13.5 \pm 2.03$ \\
\hline & & & 5 & $283.0 \pm 34.3$ & $13.7 \pm 1.5$ & $3.92 \pm 1.17$ & $4.13 \pm 0.33$ & $29.15 \pm 3.6$ \\
\hline & & & 10 & $405.7 \pm 163.5$ & $13.7 \pm 1.5$ & $4.32 \pm 0.65$ & $4.13 \pm 0.33$ & $41.9 \pm 17.1$ \\
\hline & & & 20 & $963.5 \pm 84.8$ & $13.7 \pm 1.5$ & $6.93 \pm 0.19$ & $4.13 \pm 0.33$ & $99.9 \pm 8.87$ \\
\hline \multirow[t]{2}{*}{ CEM } & $\operatorname{sgc4}$ & sgc4-poly $T_{10}-B$ & 5 & $54.5 \pm 1.78$ & $20.85 \pm 1.68$ & $3.92 \pm 1.17$ & $4.13 \pm 0.33$ & $3.17 \pm 0.43$ \\
\hline & & & 10 & 1156 & $20.85 \pm 1.68$ & $4.32 \pm 0.65$ & $4.13 \pm 0.33$ & 68.8 \\
\hline HeLa & sgc8 & sgc8-poly $T_{10}-B$ & 10 & 80.6 & 10.77 & $3.54 \pm 0$ & $4.37 \pm 0$ & 11.5 \\
\hline Ramos & TDO5 & TDO5- poly $T_{10}-B$ & 10 & $1038 \pm 202$ & $60.1 \pm 30.0$ & $7.00 \pm 4.7$ & $5.16 \pm 0$ & $18.8 \pm 6.25$ \\
\hline $\mathrm{HL}-60$ & $\mathrm{KH} 1 \mathrm{C} 12$ & $\mathrm{KH} 1 \mathrm{C} 12-\mathrm{PEG}_{18}-\mathrm{B}$ & 10 & $149.2 \pm 41.4$ & $11.06 \pm 3.4$ & $4.02 \pm 0.67$ & $3.10 \pm 0.31$ & $18.24 \pm 5.2$ \\
\hline HL-60 & KK1B10 & KK1B10-PEG $18-$-B & 10 & $120.1 \pm 19.0$ & $6.67 \pm 2.2$ & $4.02 \pm 0.67$ & $3.10 \pm 0.31$ & $31.36 \pm 5.1$ \\
\hline
\end{tabular}

Aptamer concentration: $250 \mathrm{nmol} / \mathrm{L}$

${ }^{\mathrm{b}} 2.5,5,10$, and $20 \mu \mathrm{g}$ are equivalent to $8 \times 10^{9}, 1.6 \times 10^{10}, 3.2 \times 10^{10}$, and $6.4 \times 10^{10}$ nanoparticles and to a final concentration in the incubation media of around 70, 140, 280, and $560 \mathrm{pmol} / \mathrm{L}$

' Signal enhancement determined following the equation: $S E=\{F($ Apt/NP)-F(NP) $\} /\{F($ Apt-FITC)-F(Lib-FITC) $\}$

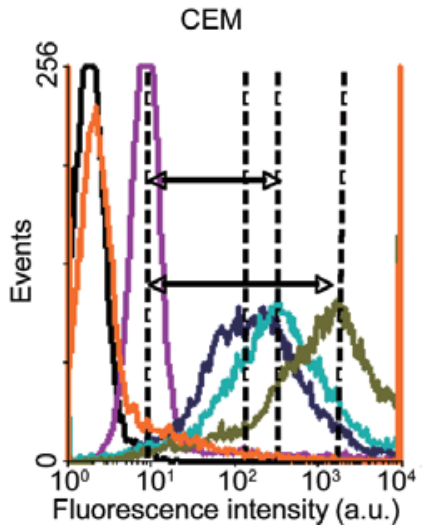

(a)

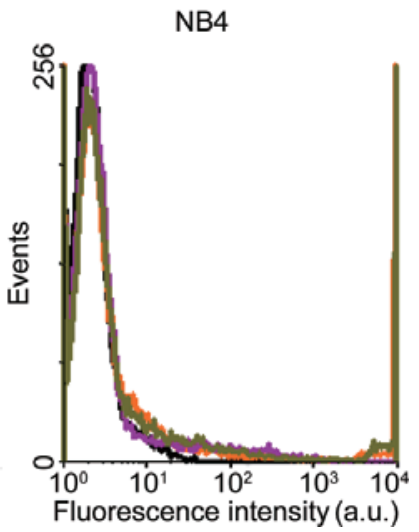

(b)
Figure 3 Flow cytometry analyses using sgc8-poly $T_{10}$-biotin aptamer and dye-doped silica NPs. (a) CEM cells (target) and (b) NB4 cells (negative cells). The black curve represents cells only, the purple curve represents cells incubated with aptamer-FITC (125 nmol/L), and the orange curve represents cells and NPs $(20 \mu \mathrm{g})$ in the absence of specific aptamer. The other curves represent incubation with aptamer first ( $125 \mathrm{nmol} / \mathrm{L}$ ) followed by different amounts of the NPs (dark blue curve: $2.5 \mu \mathrm{g}\left(8 \times 10^{9} \mathrm{NPs}, 70 \mathrm{pmol} / \mathrm{L}\right)$; cyan curve: $5 \mu \mathrm{g}\left(1.6 \times 10^{10}\right.$ NPs, $140 \mathrm{pmol} / \mathrm{L})$; brown curve: $\left.20 \mu \mathrm{g}\left(6.4 \times 10^{10} \mathrm{NPs}, 560 \mathrm{pmol} / \mathrm{L}\right)\right)$. To simplify, in the negative cells, only the curve corresponding to the highest amount of NPs (in brown) is shown the PEG linker, followed by the layer of neutravidin, offers a surface modification that efficiently and completely minimizes the nonspecific adsorption. It is worth mentioning that under the conditions at which the experiments were performed, with incubation periods of $20 \mathrm{~min}$ at $4{ }^{\circ} \mathrm{C}$ with the aptamer and 10 min with the NPs at the same temperature, no internalization should be expected either of the aptamer, as previously reported [26], or the NPs. The specificity of the system was also evaluated by confocal microscopy (see Fig. 4). The same aptamer sequence was used for these experiments, but labeled with Cy5 dye at the 5 -end $\left(\mathrm{Cy} 5\right.$-sgc8-poly $\left.\mathrm{T}_{10}-\mathrm{B}\right)$ in order to first monitor the binding of the aptamer prior to the addition of the NPs. As shown in Fig. 4, the aptamer binds specifically to the target cells (blue channel, Fig. 4(d)), and, after the washing step to remove the unbound material, the added NP(FITC)NA (NA = neutravidin) binds specifically to the biotin groups of the aptamer (green channel) (Fig. 4(d)). On the other hand, when no aptamer was added to the target cell, the incubation of the target cells with 


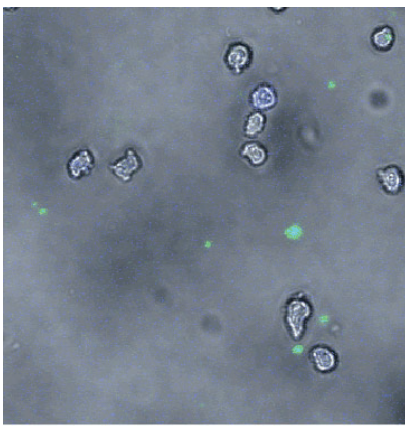

(a)

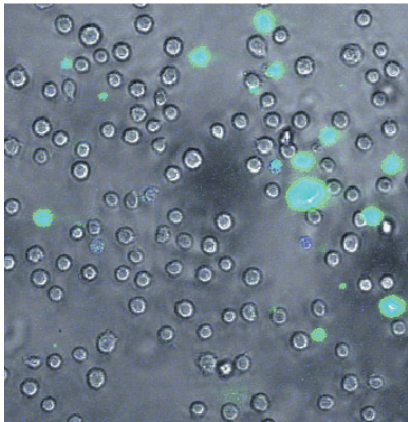

(c)

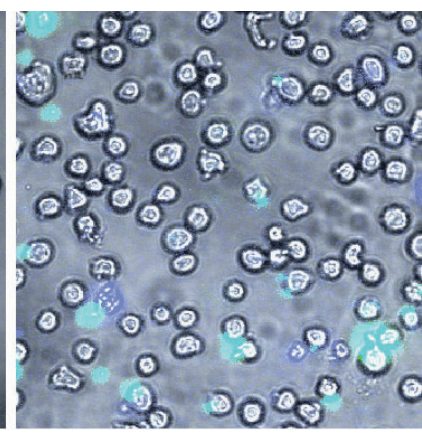

(b)

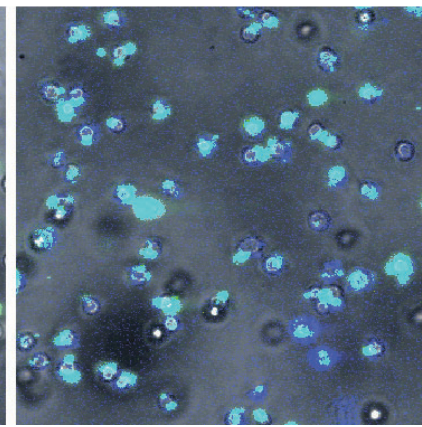

(d)
Figure 4 Fluorescence images of control (NB4) and target (CEM) cells with sgc8 aptamer and NPs (FITC): (a) NB4 cells with NPs in the absence of aptamer; (b) NB4 cells incubated with Cy5-sgc8-poly $T_{10}-B$ followed by incubation with NP(FITC)-NA; (c) CEM cells with NPS in the absence of aptamer; (d) CEM cells incubated with Cy5-sgc8poly $_{10}-\mathrm{B}$ followed by incubation with NP(FITC)-NA. The blue channel corresponds to the Cy5 signal, and the green channel corresponds to the FITC signal

NP(FITC)-NA only showed the presence of some residual aggregates of NPs. Since this did not involve overlapping with the cells, low nonspecific binding was also confirmed (Fig. 4(c)). When a negative cell line was tested, neither the aptamer nor the NPs were bound to the cells (Figs. 4(a) and 4(b)). This finding also confirms that the high level of specificity achieved with this configuration results from (1) the use of a highly specific aptamer and (2) the use of a bright nanoprobe which successfully and selectively binds only to the targeted cells, which are already present in the specific biotinylated ligand on the surface.

In order to demonstrate the feasibility of using this system for different cell lines, the NPs were further tested with different available aptamers previously selected for several cancer cell lines. In all cases, a moderate mean fluorescence was obtained with single dye-labeled aptamers under conditions where the surface receptors were saturated, whereas a signal enhancement of around one order of magnitude was achieved either for both suspension cells (CEM, Ramos, HL-60 cells) and for adherent cells (HeLa cells) (Table 2, and Fig, S-1 in the Electronic Supplementary Material (ESM)). Similar results were obtained using NP(Rubpy)-NA NPs (see Fig. S-2 in the ESM) when they were tested with different aptamer/cell combinations (sgc8/CEM and TDO5/Ramos) under the same conditions, with mean fluorescence values of $886 \pm 206.2$ and 509 \pm 73.3 , respectively, when $10 \mu \mathrm{g}$ of $\mathrm{NP}$ (Rubpy) was used. This result shows that, irrespective of the initial synthesis procedure chosen, similar behavior can be obtained, as long as NPs are prepared with the same final surface modification as defined above.

Although aptamers are now gaining acceptance as target recognition molecules, antibodies are still more widely used for flow cytometry measurements in disease diagnosis. As opposed to aptamers, which are negatively charged and thus more prone to nonspecific interactions, proteins, like antibodies, are usually neutral, conferring a more suitable and convenient environment for specific interaction with cells and also with NPs. Hence, in order to evaluate their behavior in combination with the NPs developed here, an antibody against the receptor protein tyrosine kinase 7 (PTK7) was used as an illustrative example. PTK7 has also been identified as the cell membrane protein target for $\mathrm{sgc} 8$ aptamer [25]. Thus, biotinylated monoclonal $\alpha$-PTK7 antibody was conjugated to NP(FITC)-NA and NP(Rubpy)NA NPs, and the fluorescence signal was compared with single dye-labeled $\alpha$-PTK7-Alexa 488. Although slightly brighter, Alexa 488 has similar excitation and emission spectra to NP(FITC); therefore, Alexa 488 can also be monitored in the same channel. As shown in Fig. 5, when compared with fluorophore-labeled antibodies, which are bound to $5-10$ fluorophores according to commercial specifications, a higher fluorescence signal was achieved with the NPs. The overall enhancement of the NPs reached two orders of magnitude (around 108 times, Figs. 5(a) and 5(b)) compared with the dye-labeled antibody. In a manner similar to aptamer-conjugated NPs, antibodyconjugated NPs were tested on cells without PTK7, 


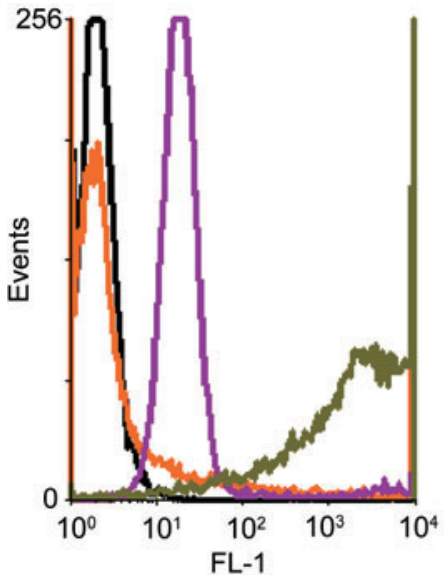

(a)

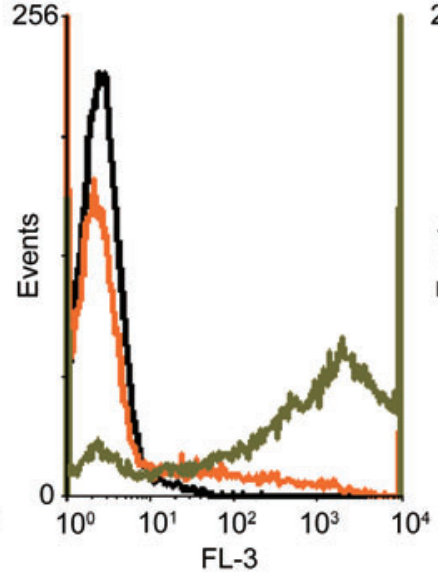

(b)

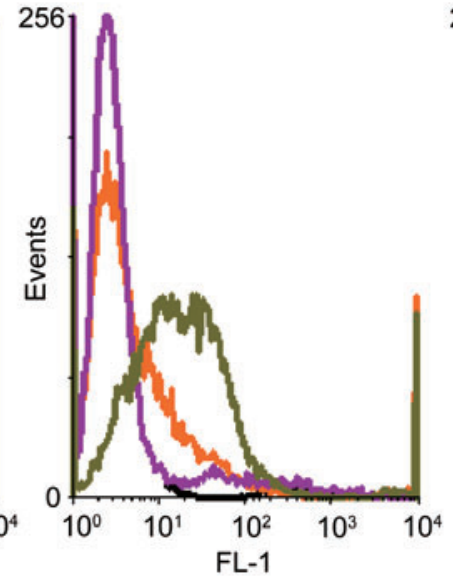

(c)

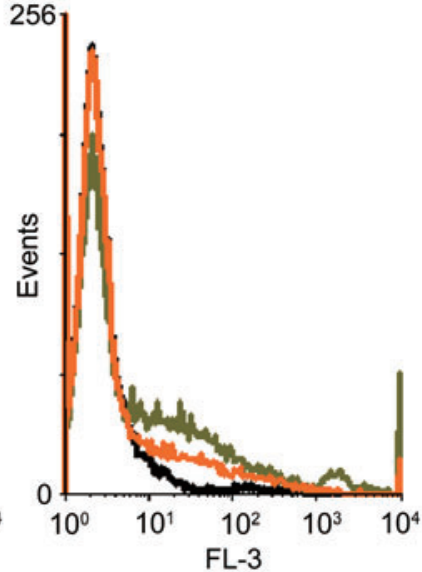

(d)

Figure 5 Flow cytometry analyses using anti-PTK7-biotin monoclonal antibody and dye-doped silica NPs with target and negative cells: (a) CEM target cells with NP(FITC)-NA; (b) CEM cells with NP(Rubpy)-NA; (c) NB4 cells (negative cell) with NP(FITC)-NA; (d) NB4 cells (negative cell) with NP(Rubpy)-NA. The black curve represents cells only; the orange curve represents cells and NPs $\left(10 \mu \mathrm{g}, 3.2 \times 10^{10} \mathrm{NPs}, 280 \mathrm{pmol} / \mathrm{L}\right)$ in the absence of antibody; the purple curve represents incubation with a-PTK7-Alexa $488(7 \mu \mathrm{L}, 600 \mathrm{nmol} / \mathrm{L})$; the brown curve represents incubation with a -PTK7-Biotin (7 nmol/L) followed by the NPs $\left(10 \mu \mathrm{g}, 3.2 \times 10^{10} \mathrm{NPs}, 280 \mathrm{pmol} / \mathrm{L}\right)$

and only a minimum shift was observed (Figs. 5(c) and 5(d)).

Since target cells in a cell mixture can be at a low concentration, we wanted to test the signal strength of NPs against a more complex system based on a mixture of target and control cells. To accomplish this, CEM cells and sgc8 aptamer were chosen as the target and specific ligand, respectively. Ramos cells, which are not recognized by sgc 8 aptamer, were added as negative cell line at different ratios. When labeled with a single fluorophore, the signal achieved by the aptamer itself was not particularly high (mean fluorescence around 13). Therefore, as shown in Fig. 6 , the low fluorescence signal prevented the single fluorophore-labeled sgc8-FITC incubated with cells from discriminating between the two populations based on differences in fluorescence (only one peak appears with an average mean fluorescence). As the amount of target cells decreases (Fig. 6, from Fig.6(a) to Fig.6(g)), the peak shifts back to lower fluorescence (from 10.73 to 5.27), finally reaching a value similar to the background from the pure cells. On the other hand, when using the NP procedure, the target cells were observed to be clearly differentiated from the negative cell population, even at a very low concentration (around 20\% of target and $80 \%$ of negative cells, as shown in Fig. 6(e)). This result highlights the benefit of using our high-amplification fluorescent NPs for monitoring the types of rare events otherwise undetectable with the conventional labels used in flow cytometry.

One of the advantages of using flow cytometry is the ability to simultaneously detect several different proteins conjugated to different and distinguishable fluorophores. As silica NPs can be doped with practically any desired dye, this type of multiplexing is possible. A preliminary study of the effectiveness of this strategy was performed by coupling sgc8-poly $\mathrm{T}_{10}$-biotin and TDO5-poly $\mathrm{T}_{10^{-}}$ biotin aptamers to NP(Rubpy)-NA and NP(FITC)NA, respectively. Thus, in this case, instead of two successive incubation steps with the cells, only one is necessary. The conjugates were then tested with the corresponding target cells and showed a fluorescence signal similar to the one obtained with the twostep incubation strategy (see Fig. S-3 in the ESM). By carefully selecting and optimizing the surface modification, we have therefore been able to extend the versatility of the fluorescent silica NPs for use in two different cell detection configurations. These results show the improved capabilities and attractive potential of these NPs for flow cytometry analysis, either as universal probes or as specifically labeled probes for multiplex detection. 


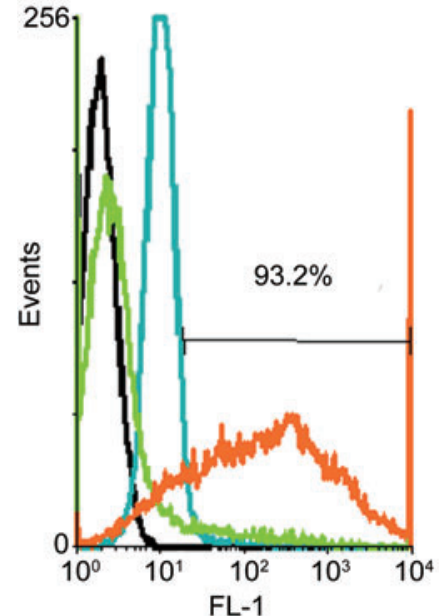

(a)

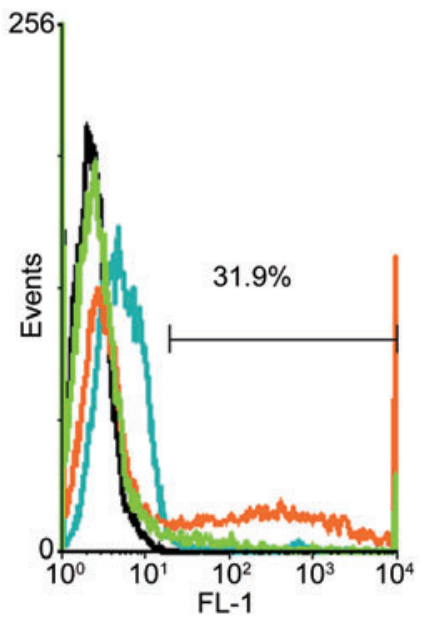

(e)

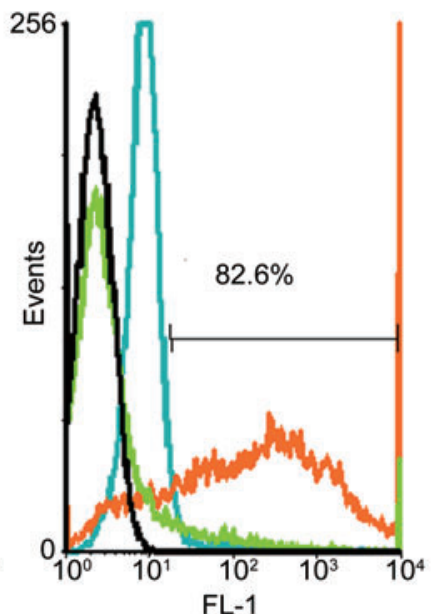

(b)

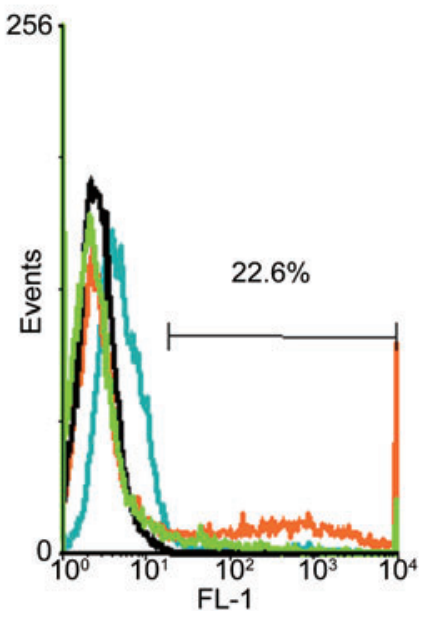

(f)

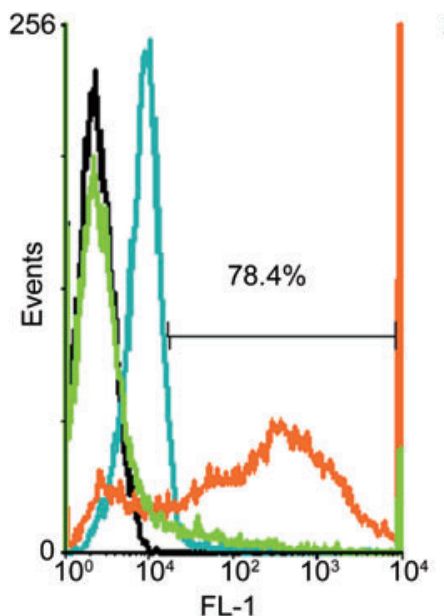

(c)

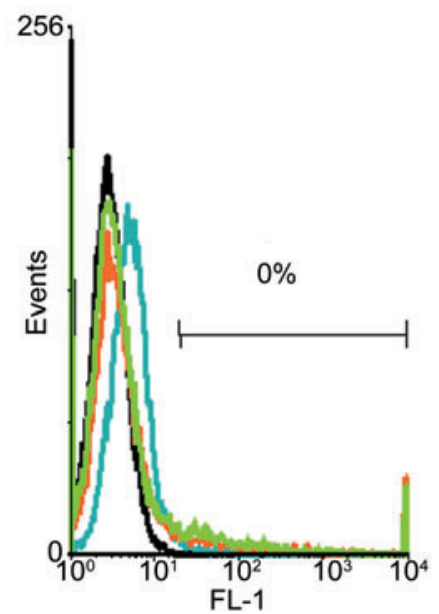

(g)

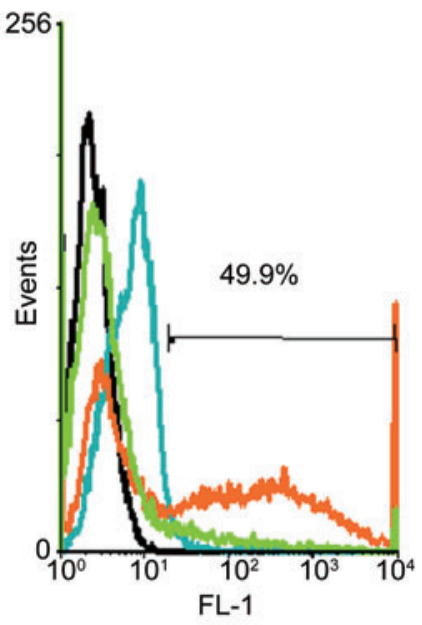

(d)

Figure 6 Flow cytometry analysis of a binary mixture of target (CEM) and negative (Ramos) cells using sgc8-poly $\mathrm{T}_{10}$-biotin aptamer and dyedoped silica NPs (NP(FITC)-NA): (a) 100\% CEM, 0\% Ramos; (b) 90\% CEM, 10\% Ramos; (c) 83\% CEM, 17\% Ramos; (d) 50\% CEM, 50\% Ramos; (e) 17\% CEM, 83\% Ramos; (f) 10\% CEM, 90\% Ramos; (g) 0\% CEM, 100\% Ramos. Black line: pure cells; green line: cells incubated with NPs in the absence of aptamer; blue line: cells incubated with sgc8-FITC; orange line: cells incubated with sgc8-polyT 10 -biotin and NPs

\section{Conclusions}

We have combined the selectivity of aptamers and antibodies with the high signal enhancement and ease of surface functionalization offered by dyedoped silica NPs to enhance the detection capabilities of conventional flow cytometry. By using NPs which trap several thousands of dye molecules in the silica matrix, a enhancement of one to two orders of magnitude can be achieved. Furthermore, the ability to prepare dye-doped NPs with virtually any of the currently available fluorophores, or even multiple dyes inside one NP [21], provides for multiplex detection of several targets simultaneously. It is true that nonspecific binding caused by both the intrinsic nature of NPs, as well as the cell membrane, stands in the way of effectively using NPs for highly sensitive analysis. However, we addressed this challenge by performing surface chemistry studies of the NPs and by surface coating the silica NPs with a PEG moiety, which increases their hydrophilicity and biocompatibility, and neutravidin, which allows the coupling of the biotinylated molecules. This surface coating procedure (1) minimizes nonspecific binding of the probes to the cell surface and (2) provides a universal and straightforward binding design easily adaptable to multiple types of biomolecules.

To demonstrate the overall viability of our NP 
system, different aptamers and target cells were tested. This method is especially promising in the case of DNA probes such as aptamers which are usually single dye-labeled. To our knowledge, this is the first time highly fluorescent silica NPs have been implemented as a fluorescent label to efficiently increase the signal in flow cytometry for the purpose of cell detection. The results shown suggest the possible value of fluorescent silica NPs not only in clinical diagnostics but also in other biological applications based on cell analysis, when sensitivity can become a limiting factor. The high signal enhancement, as well as the possibility of preparing NPs with any type of dye meeting instrument requirements, can therefore increase the potential of flow cytometry in situations where the number of target cells is small compared to the cell pool. Nevertheless, further experiments have to be performed to precisely determine the minimum number of target cells capable of being detected by flow cytometry in a mixture of cells using these fluorescent NPs.

\section{Acknowledgements}

This work was supported by the NIH National Cancer Institute, R21CA122648, ONR N00014-07-1-0982, and the State of Florida Center of Excellence. M. -C. E. acknowledges financial support from the Department d'Universitats, Recerca i Societat de la Informació de la Generalitat de Catalunya, Spain.

Electronic Supplementary Material: Supplementary material (details of determination of the fluorescence signal amplification and three figures as noted in the text) is available in the online version of this article at http://dx.doi.org/10.1007/s12274-009-9041-8 and is accessible free of charge.

\section{References}

[1] Prince, H. E.; Arens, L.; Kleinman, S. H. CD4 and CD8 subsets defined by dual-color cytofluorometry which distinguish symptomatic from asymptomatic blood donors seropositive for human immunodeficiency virus. Diagn. Clin. Immunol. 1987, 5, 188-193.
[2] Craig, F. E. Flow cytometric evaluation of B-cell lymphoid neoplasms. Clin. Lab. Med. 2007, 27, 487-512.

[3] Donnenberg, A. D.; Donnenberg, V. S. Rare-event analysis in flow cytometry. Clin. Lab. Med. 2007, 27, 627 -652 .

[4] Niemeyer, C. M. Nanoparticles, proteins, and nucleic acids: Biotechnology meets materials science. Angew. Chem. Int. Ed. 2001, 40, 4128-4158.

[5] Wang, L.; Wang, K.; Santra, S.; Zhao, X.; Hilliard, L. R.; Smith, J. E.; Wu, Y.; Tan, W. Watching silica nanoparticles glow in the biological world. Anal. Chem. 2006, 78, 646-654.

[6] Holm, B. A.; Bergey, E. J.; De T; Rodman, D. J.; Kapoor, R.; Levy, L.; Friend, C. S.; Prasad, P. N. Nanotechnology in biomedical applications. Mol. Cryst. Liq. Cryst. 2002, 374, 589-598.

[7] He, X.; Duan, J.; Wang, K.; Tan, W.; Lin, X.; He, C. A novel fluorescent label based on organic dye-doped silica nanoparticles for HepG liver cancer cell recognition. J. Nanosci. Nanotechnol. 2004, 4, 585-589.

[8] Herr, J. K.; Smith, J. E.; Medley, C. D.; Shangguan, D.; Tan, W. Aptamer-conjugated nanoparticles for selective collection and detection of cancer cells. Anal. Chem. 2006, 78, 2918-2924.

[9] Zhao, X.; Hilliard, L. R.; Mechery, S. J.; Wang, Y.; Bagwe, R. P.; Jin, S.; Tan, W. A rapid bioassay for single bacterial cell quantitation using bioconjugated nanoparticles. Proc. Natl. Acad. Sci. USA 2004, 101, 15027-15032.

[10] Shangguan, D.; Li, Y.; Tang, Z.; Cao, Z. C.; Chen, H. W.; Mallikaratchy, P.; Sefah, K.; Yang, C. J.; Tan, W. Aptamers evolved from live cells as effective molecular probes for cancer study. Proc. Natl. Acad. Sci. 2006, 103, 11838 11843

[11] Tang, Z.; Shangguan, D.; Wang, K.; Shi, H.; Sefah, K.; Mallikaratchy, P.; Chen, H. W.; Li, Y.; Tan, W. Selection of aptamers for molecular recognition and characterization of cancer cells. Anal. Chem. 2007, 79, 4900-4907.

[12] Chattopadhyay, P. K.; Price, D. A.; Harper, T. F.; Betts, M. R.; Yu, J.; Gostick, E.; Perfetto, S. P.; Goepfert, P.; Koup, R. A.; De Rosa, S. C.; Bruchez, M. P.; Roederer, M. Quantum dot semiconductor nanocrystals for immunophenotyping by polychromatic flow cytometry. Nat. Med. 2006, 12, 972-977.

[13] Stöber, W.; Fink, A.; Bohn, E. Controlled growth of monodisperse silica spheres in the micron size range. $J$. Colloid Interf. Sci. 1968, 26, 62-69. 
[14] Bagwe, R. P.; Zhao, X.; Tan, W. Bioconjugated luminescent nanoparticles for biological applications. J. Disper. Sci. Technol. 2003, 24, 453-464.

[15] Bagwe, R. P.; Hilliard, L. R.; Tan, W. Surface modification of silica nanoparticles to reduce aggregation and nonspecific binding. Langmuir 2006, 22, 4357-4362.

[16] Hilliard, L. R.; Zhao, X.; Tan, W. Immobilization of oligonucleotides onto silica nanoparticles for DNA hybridization studies. Anal. Chim. Acta 2002, 470, 5156.

[17] Xu, H.; Yan, F.; Monson, E. E.; Kopelman, R. Roomtemperature preparation and characterization of poly(ethylene glycol)-coated silica nanoparticles for biomedical applications. J. Biomed. Mater. Res. 2003, 66A, 870-879.

[18] Gao, X.; Cui, Y.; Levenson, R. M.; Chung, L. W. K.; Shuming, N. In vivo cancer targeting and imaging with semiconductor quantum dots. Nat. Biotechnol. 2004, 22, 969-976.

[19] Liu, Y.; Shipton, M. K.; Ryan, J.; Kaufman, E. D.; Franzen, S.; Feldheim, D. L. Synthesis, stability, and cellular internalization of gold nanoparticles containing mixed peptide-poly(ethylene glycol) monolayers. Anal. Chem.
2007, 79, 2221-2229.

[20] van Blaaderen, A.; Vrij, A. Synthesis and characterization of colloidal dispersions of fluorescent, monodisperse silica spheres. Langmuir 1992, 8, 2921-2931.

[21] Wang, L.; Tan, W. Multicolor FRET silica nanoparticles by single wavelength excitation. Nano Lett. 2006, 6, 84-88.

[22] Santra, S.; Wang, K.; Tapec, R.; Tan, W. Development of novel dye-doped silica nanoparticles for biomarker application. J. Biomed. Opt. 2001, 6, 160-166.

[23] Brussaard, C. P. D.; Marie, D.; Bratbak, G. Flow cytometric detection of viruses. J. Virol. Methods 2000, 85, 175-182.

[24] Shangguan, D.; Cao, Z. C.; Li, Y.; Tan, W. Aptamers evolved from cultured cancer cells reveal molecular differences of cancer cells in patient samples. Clin. Chem. 2007, 53, 1153-1155.

[25] Shangguan, D.; Cao, Z.; Meng, L.; Mallikaratchy, P.; Sefah, K.; Wang, H.; Li, Y.; Tan, W. Cell-specific aptamer probes for membrane protein elucidation in cancer cells. J. Proteome Res. 2008, 7, 2133-2139.

[26] Xiao, Z.; Shangguan, D.; Cao, Z.; Fang, X.; Tan, W. Cellspecific internalization study of an aptamer from whole cell selection. Chem. Eur. J. 2008, 14, 1769-1775. 